\title{
Macroecologia de mamíferos neotropicais com ocorrência no Cerrado
}

\author{
Cleiber Marques Vieira ${ }^{1}$ \\ José Alexandre Felizola Diniz-Filho ${ }^{2}$
}

\begin{abstract}
Macroecology of neotropical mammals with occurrence in the brazilian "Cerrado". The macroecological approach has been used recently to analyze correlations between ecological variables in large taxonomic groups, at continental scales. A positive relationship between body weight and geographic range size has been described as a poligonal space envelope form, that can be explained by ecological and evolutionary constraints. However, these variables can be disturbed by spatial and phylogenetic autocorrelation effects. In this work, the relationship between body weight and geographic range size was analyzed for 80 species of Neotropical mammals present in the Brazilian "cerrado". Spatial and taxonomic effects were tested using a linear trend surface analysis and an ANOVA (at level of order), combined in a generalized model. Around $61 \%$ of variation in geographic range size and $69 \%$ of variation in body weight in mammals of the "cerrado" can be explained simultaneously by spatial and taxonomic effects. The analysis of residuals of the generalized model showed that positive correlation between variables persist even after removing these effects. Thus, variation and covariation of traits in the mammals of the brazilian "cerrado" agree with the general macroecological pattern proposed for another taxonomic groups such as mammals, birds and snakes worldwilde.

KEY WORDS. Macroecology, "Cerrado" mammals, geographic range size, ecological and evolutionary constraints, spatial and phylogenetic autocorrelation
\end{abstract}

A macroecologia analisa as relações entre variáveis ecológicas mensuradas em escalas grandes de tempo e espaço (BROWN \& MAURER 1987, 1989; LEVIN 1992; BROWN 1995). A análise de variáveis macroecológicas, tais como o tamanho do corpo, área de distribuição e densidade populacional local das espécies, mensuradas em grandes grupos taxonômicos em escalas continentais, pode revelar processos ecológicos e evolutivos que participam na estruturação das comunidades (LABARBERA 1989; PETERS 1993; RickleFs \& SCHLUTER 1993; BROWN 1995).

Dentre as principais relações macroecológicas, a obtida entre o tamanho do corpo (definida pela massa corpórea) e a área de distribuição das espécies tem sido descrita para diversos grupos taxonômicos, tais como insetos, peixes, aves e mamíferos, principalmente da América do Norte (BROWN \& MAURER 1987, 1989; GASTON \& LAWTON 1988a,b; TAYLOR \& GOTELli 1994; GASTON \& BLACKBURN

1) Departamento de Biologia, Centro de Ciências Exatas e Tecnológicas, Universidade Estadual de Goiás. Caixa Postal 459, 75110-380 Anápolis, Goiás, Brasil.

E-mail: cleibermv@uol.com.br

2) Departamento de Biologia Geral, Instituto de Ciências Biológicas, Universidade Federal de Goiás. Caixa Postal 131, 74001-970 Goiânia, Goiás, Brasil. E-mail: diniz@icb1.ufg.br 
1996a; Diniz-FilHo \& Fowler 1998). GASTON \& BLACKBURN (1996b) discutem pelo menos quatro mecanismos que podem explicar a correlação positiva entre o tamanho do corpo e a área de distribuição mínima:

1) Hipótese do tamanho mínimo viável da população. Na qual a relação positiva é representada por uma forma poligonal triangular, definida por linhas de restrição (constraints) absolutas (tamanho mínimo do corpo e área máxima de distribuição) e por uma linha de restrição probabilística, na qual a área mínima de distribuição, abaixo e acima do tamanho modal, estaria correlacionada com o tamanho do corpo. Essa linha representaria uma distribuição mínima capaz de manter uma densidade local que minimize a tendência a extinção estocástica (BROWN \& MAURER 1987, 1989);

2) Hipótese da exploração do potencial máximo da área de distribuição. Na qual a área mínima tende a aumentar com o crescimento do tamanho corpóreo pois, em média, espécies grandes apresentam dispersão mais rápida e eficaz se comparadas com espécies pequenas. Além disso, espécies maiores devem ter ocupado distribuições mais extensas por apresentarem mais tempo de evolução, havendo assim um efeito de inércia filogenética nas variáveis;

3) Hipótese da homeostase e heterogeneidade ambiental. Na qual espécies grandes tendem a apresentar uma maior homeostase, se comparadas àquelas de menor tamanho, ou seja, elas podem sobreviver em condições ambientais mais heterogêneas. Logo, espécies grandes devem suportar melhor a heterogeneidade de habitats observada em distribuições extensas, quando comparadas com espécies pequenas;

4) Hipótese dos gradientes latitudinais. Na qual a área de distribuição e tamanho do corpo apresentam uma correlação positiva com a latitude, concordando, respectivamente, com as regras de Rapoport e Bergmann. Isso gera uma correlação aparente entre essas variáveis, mas que não implica em uma relação ecológica ou adaptativa entre elas.

Por outro lado, GASTON \& LAWTON (1988a,b) sugerem uma correlação negativa entre o tamanho do corpo e a área de distribuição. Nesse modelo, animais com tamanho do corpo pequeno, como os insetos, apresentam grandes áreas de distribuição, pois como a taxa de crescimento populacional intrínseca decresce com o aumento do tamanho do corpo, espécies pequenas têm uma capacidade de colonização maior, alcançando distribuições maiores que aquelas com tamanho grande.

Contudo, na avaliação empírica desses modelos, é importante notar que as relações entre variáveis macroecológicas, tais como o tamanho do corpo e a área de distribuição, são mensuradas a partir de um grande número de espécies, que estão interligadas por um ancestral comum ao longo de uma filogenia, e distribuídas continuamente no espaço geográfico (HARVEY \& PAGEL 1992). Assim, essas relações podem estar sendo perturbadas por efeitos de inércia filogenética (hipótese 2) e/ou por padrões espaciais (hipótese 4) (RUGGIERO 1994; GASTON \& BLACKBURN 1996a,b; BLACKBURN \& GASTON 1998; GASTON et al. 1998; BONFIM et al. 1998; MARQUeT \& TAPER 1998; SANT'ANA \& DINIZ-FILho 1999). Para a obtenção de 
correlações não-viesadas entre as variáveis analisadas, portanto, é necessário utilizar técnicas de análise comparativa filogenética e/ou espacial capazes de detectar e remover tais efeitos.

Os mamíferos estão representados por aproximadamente 600 espécies na região neotropical (incluindo os mamíferos voadores e mamíferos aquáticos), distribuídas em 11 ordens diferentes (EISENBERG 1989; REDFORD \& EISENBERG 1992; ALHO 1993; WILSON \& REEDER 1993; EMMONS 1997). Eles apresentam uma enorme variação morfológica e adaptativa, o que os torna de especial interesse para análises macroecológicas. Trabalhos correlatos analisaram padrões macroecológicos de mamíferos com ampla distribuição geográfica na América do Norte e Europa (BROWN \& MAURER 1987, 1989). Entretanto, poucos trabalhos com essa abordagem analisam os mamíferos na América do Sul (ARITA et al. 1990; RUGGIERO 1994; DiNIZ-FILHO \& BALESTRA 1998).

O primeiro objetivo desse trabalho foi avaliar se a relação entre o tamanho do corpo e área de distribuição, para os mamíferos com ocorrência no cerrado brasileiro, apresenta um padrão similar àquele observado para outros grupos taxonômicos. O segundo foi testar se essa correlação ocorre em resposta à processos ecológicos e evolutivos subjacentes ou se é apenas um artefato dos efeitos espaciais e/ou filogenéticos.

\section{MATERIAL E MÉTODOS}

Para a análise das relações macroecológicas dos mamíferos foram utilizadas duas variáveis: o tamanho médio do corpo, expresso pelo peso médio do corpo, e a área de distribuição da espécie. Foram coletados dados de tamanho do corpo e área de distribuição de 80 espécies de mamíferos terrestres (não-voadores) com ocorrência na região do cerrado brasileiro (Tab. I). Essas espécies estão distribuídas em oito Ordens diferentes (Marsupialia ou Didelphimorphia, Edentata ou Xenarthra, Rodentia, Carnivora, Lagomorpha, Perissodactyla, Artiodactyla e Primates).

Os dados de peso foram extraídos da literatura (EISENBERG 1989; REDFORD \& EISENBERG 1992; FONSECA 1996; EMMONS 1997) e, para corrigir os efeitos de alometria e heterocedasticidade, foram logaritmizados antes das análises (BROWN \& MAURER 1987, 1989; SOKAL \& ROHLF 1995; GASTON 1996). As espécies selecionadas para essa análise seguiram os critérios de: 1) disponibilidade de dados na literatura para as variáveis analisadas; 2 ) apresentarem pelo menos uma quadrícula da sua área de distribuição com ocorrência dentro da área do cerrado e; 3) não apresentarem muitos problemas em relação a sua taxonomia alfa (WILSON \& REEDER 1993).

As áreas de distribuição foram definidas pela área de extensão de ocorrência total da espécie (GASTON 1991, 1996) e foram compiladas a partir de mapas de distribuição apresentados em diversos trabalhos (MOOJEN 1952; MARES \& GENOWAYS 1982; EISENBERG 1989; REDFORD \& EISENBERG 1992; WilsON \& REEDER 1993; EMMONS 1997). Para determinar o tamanho da área de distribuição das espécies foi utilizado o método das quadrículas (GASTON 1996). Para isso, todos os mapas foram redesenhados numa escala padronizada (escala $=1: 40.000 .000)$ de um mapa das Américas (CAMPBEll \& LAMAR 1989) coberto por uma malha de quadrículas com 156,5 km de lado $\left(24.492,25 \mathrm{~km}^{2}\right.$ ) (Fig. 1). 


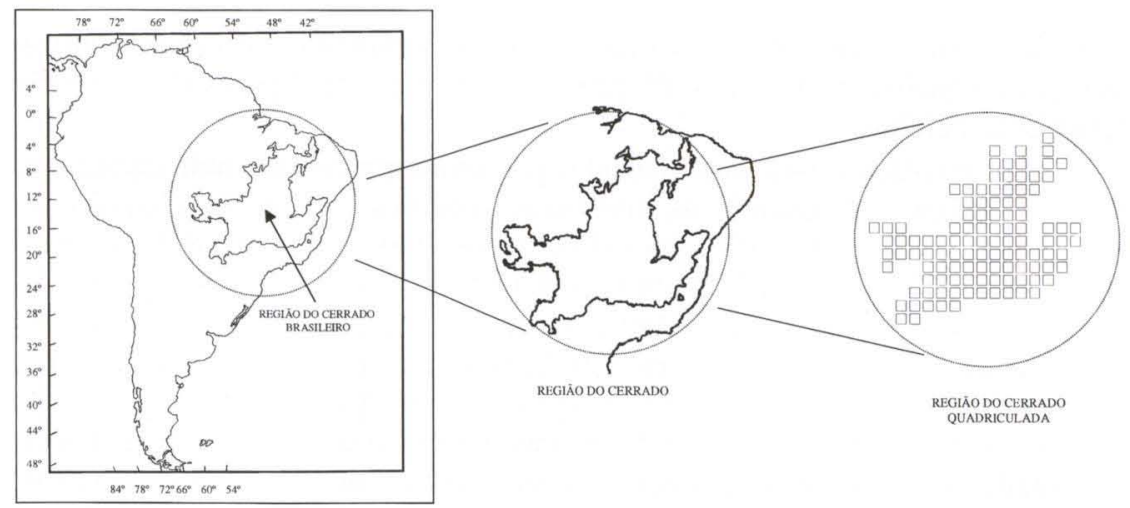

Fig. 1. Mapa base da América do Sul (adaptado de CAMPBELL \& LAMAR 1989), área do cerrado em detalhe e área do cerrado representada pela malha de quadriculas com $156.5 \mathrm{Km}$ de lado.

Foram analisadas as distribuições de freqüência para as duas variáveis macroecológicas. Como muitos problemas vêm sendo discutidos em relação ao ajuste de padrões de distribuição via inspeção visual, foram usados testes estatísticos convencionais para esse tipo de análise, como o de Lilliefors, que testa o ajuste da distribuição analisada contra a distribuição normal esperada (TOKESHI 1993).

Os padrões espaciais foram analisados para cada uma das variáveis. Para isso, foi utilizado inicialmente o método dos pontos médios (midpoints), pareandose os pontos médios de latitude e longitude das áreas de distribuição das espécies analisadas com os valores observados para cada uma das variáveis.

Para detectar os possíveis efeitos espaciais nos dados foram utilizadas análises de autocorrelação espacial (SOKAL \& ODEN 1978a,b; LEGENDRE \& FORTIN 1989; LEGENDRE 1993). Correlogramas foram construídos com base nos índices I de Moran, calculados em intervalos constantes em 10 classes de distância (ver figura 4). O índice I de Moran, que varia entre 1.0 e -1.0, é dado pela seguinte fórmula:

$$
I=(n / K) \sum_{i} \sum_{j} W_{i j} Z_{i} Z_{j} / \sum_{i} Z_{i}^{2}
$$

Onde, $\mathbf{n}$ é o número de espécies, $\mathbf{W}_{\mathbf{i j}}$ é o elemento $\mathbf{i}, \mathbf{j}$ da matriz de conectividade W ( 0 ou 1$)$ em uma determinada classe de distância entre os centros das áreas de distribuição das espécies $\mathbf{i}$ j, $\mathbf{Z}$ é o valor da variável analisada centrada pela média e $\mathbf{K}$ é a soma dos valores da matriz W.

A magnitude e o nível de significância do índice I de Moran indicam a similaridade das espécies em relação aos seus pontos médios em uma determinada classe de distância. Assim, uma classe de distância com o índice I de Moran significativo e positivo indica, para aquele intervalo de distância espacial, espécies cujas áreas de distribuição geográfica ou tamanhos do corpo são mais similares entre si do que com outras espécies (LEGENDRE \& ForTIN 1989; LEGENDRE 1993; BONFIM et al. 1998). A significância do correlograma como um todo foi testada através do critério de Bonferroni (ODEN 1984). 
Tabela I. Relação de 80 espécies de mamíferos com ocorrência no cerrado brasileiro, acompanhada do peso médio do corpo e da área de distribuição geográfica para cada espécie (as áreas estão estimadas pelo número de quadrículas de 156,5 $\mathrm{Km}$ de lado).

\begin{tabular}{|c|c|c|}
\hline \multirow[t]{2}{*}{ Ordens de Mamiferos } & \multicolumn{2}{|c|}{ Variáveis macroecológicas } \\
\hline & Peso (g) & Área \\
\hline \multicolumn{3}{|l|}{ Didelphimorphia (Marsupialia) } \\
\hline Didelphis albiventris & 1250 & 307 \\
\hline Marmosa agilis & 30 & 122 \\
\hline Marmosa murina & 32 & 365 \\
\hline Marmosa cinerea & 105 & 340 \\
\hline Monodelphis americana & 29 & 45 \\
\hline Monodelphis brevicaudata & 84 & 163 \\
\hline Monodelphis domestica & 67 & 125 \\
\hline Monodelphis kunzi & 20 & 17 \\
\hline Monodelphis umbristriatata & 45 & 15 \\
\hline Caluromys lanatus & 356 & 236 \\
\hline Caluromys philander & 170 & 180 \\
\hline Lutreolina crassicaudata & 537 & 74 \\
\hline Chironectes minimus & 665 & 202 \\
\hline Philander opossum & 360 & 345 \\
\hline Metachiurus nudicaudatus & 280 & 365 \\
\hline \multicolumn{3}{|l|}{ Xenarthra (Edentata) } \\
\hline Myrmecophaga tridactyla & 30500 & 550 \\
\hline Tamandua tetradactyla & 5200 & 546 \\
\hline Dasypus septemcinctus & 1500 & 242 \\
\hline Dasypus novemcinctus & 3650 & 746 \\
\hline Euphractus sexcinctus & 5400 & 277 \\
\hline Cabassous unicinctus & 3200 & 447 \\
\hline Tolypeutes trincinctus & 1530 & 63 \\
\hline Priodontes maximus & 26800 & 462 \\
\hline \multicolumn{3}{|l|}{ Rodentia } \\
\hline Calomys callosus & 31 & 109 \\
\hline Calomys tener & 20 & 12 \\
\hline Holocchilus brasiliensis & 280 & 106 \\
\hline Kunsia fronto & 230 & 18 \\
\hline Nectomys squamipes & 249 & 470 \\
\hline Oryzomys concolor & 63 & 511 \\
\hline Oryzomys lamia & 58 & 54 \\
\hline Oryzomys ratticeps & 144 & 81 \\
\hline Oryzomys oniscus (= capito) & 59 & 483 \\
\hline Oyzomys subflavus & 92 & 53 \\
\hline Oligoryzomys eliurus & 21 & 136 \\
\hline Oligoryzomys chacoensis & 31 & 110 \\
\hline Oxymycterus roberti & 83 & 10 \\
\hline Rhipidomys mascatalis & 80 & 170 \\
\hline Akodon cursor & 38 & 131 \\
\hline Bolomys lasiurus & 35 & 64 \\
\hline Wiedomys pyrrhorhinos & 45 & $\begin{array}{c}144 \\
\text { Cont }\end{array}$ \\
\hline
\end{tabular}


Tabela I. Continuação.

\begin{tabular}{|c|c|c|}
\hline \multirow[t]{2}{*}{ Ordens de Mamíferos } & \multicolumn{2}{|c|}{ Variáveis macroecológicas } \\
\hline & Peso $(\mathrm{g})$ & Área \\
\hline Cavia aperea & 549 & 176 \\
\hline Galea spixii & 400 & 223 \\
\hline Hydrochaeris hydrochaeris & 50000 & 602 \\
\hline Agouti paca & 8227 & 589 \\
\hline Dasyprocta azarae & 2800 & 89 \\
\hline Carterdon sulcidens & 180 & 18 \\
\hline Clyomys laticeps & 170 & 37 \\
\hline Euryzygoma guiara & 188 & 72 \\
\hline Proechimys longicaudatus & 210 & 153 \\
\hline Thrichomys apereoides & 339 & 105 \\
\hline Coendou prehensilis & 3360 & 339 \\
\hline \multicolumn{3}{|l|}{ Carnivora } \\
\hline Procyon cancrivorus & 5400 & 559 \\
\hline Nasua nasua & 5100 & 444 \\
\hline Cerdocyon (=Dusycuon) thous & 6500 & 320 \\
\hline Lycalopex velutus & 4000 & 572 \\
\hline Chrysocyon brachyurus & 22000 & 572 \\
\hline Speothos venaticus & 6000 & 257 \\
\hline Galictes cuja & 1665 & 674 \\
\hline Conepatus emistriatus & 2400 & 150 \\
\hline Pteronura brasiliensis & 29000 & 321 \\
\hline Lontra longicaudis & 5800 & 513 \\
\hline Panthera onca & 94500 & 697 \\
\hline Puma concolor & 74500 & 1040 \\
\hline Leopardus pardalis & 10000 & 657 \\
\hline Leopardus wiedii & 3220 & 612 \\
\hline Leopardus tigrinus & 2250 & 129 \\
\hline Herpailurus yaguarondi & 5000 & 646 \\
\hline \multicolumn{3}{|l|}{ Lagomorpha } \\
\hline Sylvilagus brasiliensis & 934 & 109 \\
\hline \multicolumn{3}{|l|}{ Perissodactyla } \\
\hline Tapirus terretris & 239000 & 461 \\
\hline \multicolumn{3}{|l|}{ Artiodactyla } \\
\hline Tayssu tajacu & 1900 & 650 \\
\hline Tayassu pecari & 30000 & 575 \\
\hline Mazama americana & 29000 & 572 \\
\hline Mazama gouazoubira & 16000 & 524 \\
\hline Ozotoceros bezoarticus & 35000 & 160 \\
\hline Blastocerus dichotomus & 108600 & 151 \\
\hline \multicolumn{3}{|l|}{ Primates } \\
\hline Callithrix penicillata & 290 & 58 \\
\hline Callithrix melanura & 260 & 36 \\
\hline Callithrix jacchus & 292 & 28 \\
\hline Cebus apella & 2500 & 476 \\
\hline Alouatta caraya & 6500 & 73 \\
\hline
\end{tabular}


Os padrões espaciais significativos detectados pela análise de autocorrelação foram removidos através de uma análise de superfície de tendência. Essa técnica consiste numa análise de regressão múltipla onde as variáveis independentes são as coordenadas geográficas (latitude e longitude) ou suas extensões polinomiais.

Outro aspecto nesse tipo de análise a nível de espécie é o de que podem acontecer perturbações decorrentes também de padrões filogenéticos (CHEVERUD et al. 1985; Harvey \& PAgel 1992; Diniz-Filho \& VieIRA 1998). Diversas técnicas foram sugeridas para o controle desses efeitos inerciais, porém os métodos mais freqüentemente aplicados para esse controle não puderam ser usados com os dados utilizados neste trabalho, pois exigem, pelo menos, uma filogenia consensual. Os mamíferos representam um grupo muito heterogêneo e é natural que ainda existam várias dúvidas quanto à sua taxonomia e lacunas no conhecimento das relações filogenéticas. Entretanto, um possível efeito filogenético ao nível taxonômico da ordem pôde ser analisado utilizando-se uma Análise de Variância (ANOVA) (DINIZ-FILHO \& BALESTRA 1998).

Assim, para a remoção conjunta dos efeitos espaciais e filogenéticos, foi utilizado um modelo generalizado combinando a superficie de tendência e a ANOVA ao nível das ordens. Os resíduos desse modelo foram analisados, permitindo assim que fossem correlacionadas as variáveis macroecológicas sem a influência desses efeitos.

Todas as análises foram feitas num microcomputador PC/Pentium 200, usando os programas SAAP-Pc (Spatial Autocorrelation Analysis Program) (WARTENBERG 1989) e Systat/ Sygraph (WILKINSON 1989).

\section{RESULTADOS}

As variáveis analisadas apresentaram uma distribuição de freqüência assimétrica para os dados logaritmizados. Os dados de peso do corpo apresentaram uma distribuição assimétrica à direita (Fig. 2). A mesma assimetria, porém à esquerda, foi observada nos dados de distribuição, demonstrando assim uma tendência a um maior número de áreas de distribuição grandes (Fig. 3).

As distribuições foram testadas e mesmo depois da logaritmização, nenhuma delas apresentou um ajuste satisfatório para a distribuiçaão normal (Teste de Lilliefors, $\mathrm{P}>0,05$ ). Essas anormalidades foram observadas para grupos de mamíferos e aves da América do Norte (BROWN \& MAURER 1987, 1989; BROWN 1995).

A correlação entre as variáveis macroecológicas foi positiva e significativa $(\mathrm{r}=0,62 ; \mathrm{P}<0,05)$, revelando também um espaço em forma de envelope que sugere a existência de linhas de restrição ("constraints") ecológicas e evolutivas (Fig. 4). Entretanto, a análise de autocorrelação revelou que efeitos espaciais podem estar perturbando a distribuição das variáveis analisadas. Foram obtidos correlogramas significativos, segundo o critério de Bonferroni $(\mathrm{P}<0,05)$, tanto para o peso do corpo quanto para a área de distribuição (Fig. 5).

Um padrão espacial claro foi observado para a área de distribuição, onde os índices I de Moran significativos foram obtidos para um maior número de classes de distância. As quatro primeiras classes apresentaram índices significativos positivos. A sexta, a sétima, a oitava e a décima classes apresentaram índices I de Moran significativos negativos e somente a quinta e nona não apresentaram índices significativos. 


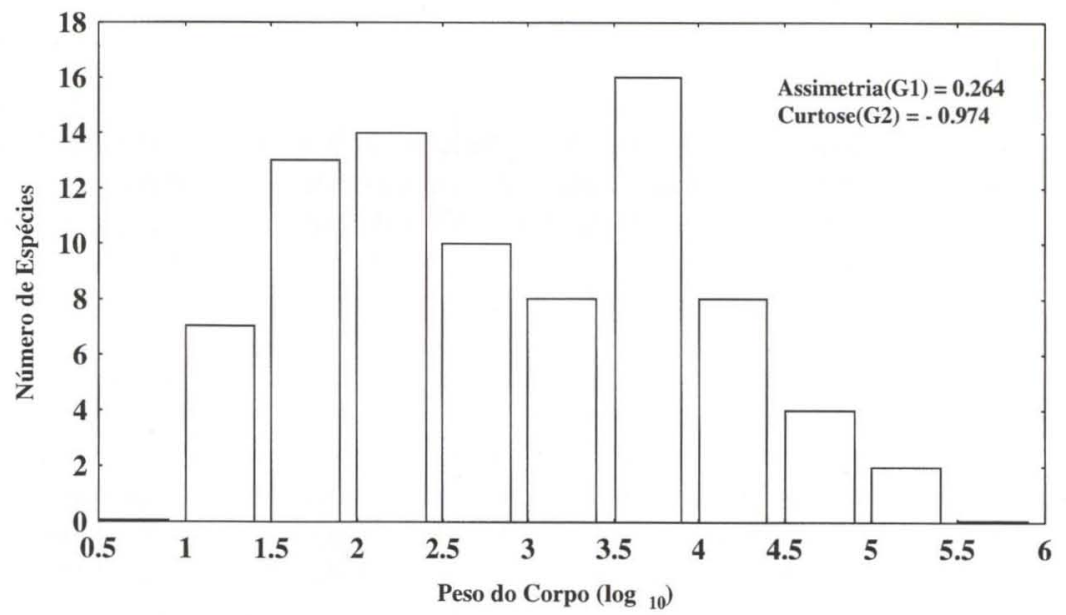

Fig. 2. Distribuição do peso do corpo logaritmizado, para 80 espécies de mamíferos com ocorrência no cerrado brasileiro.

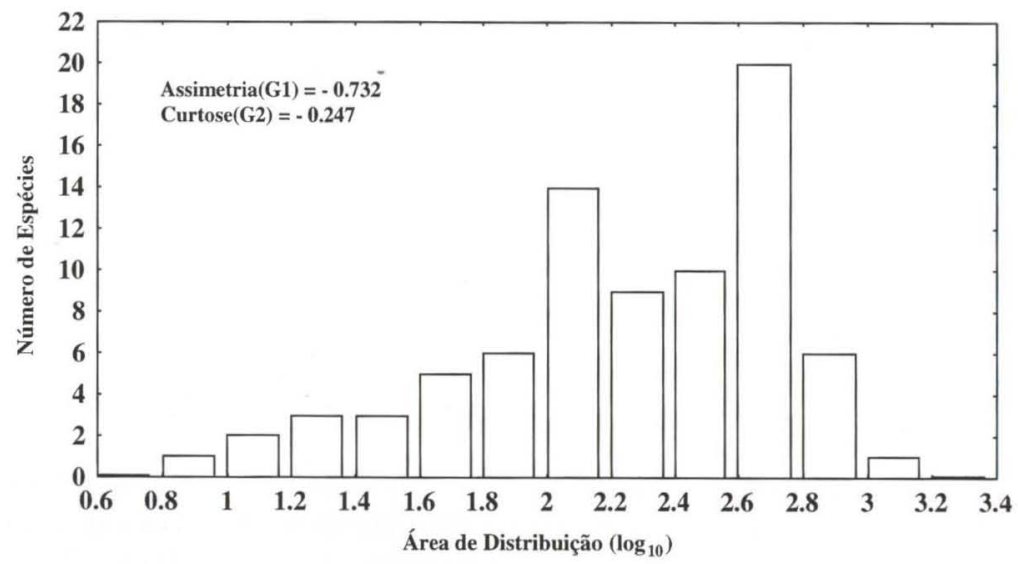

Fig. 3. Lıstribuição da área de distribuição geográfica logaritimizada, para 80 espécies de mamíferos com ocorrência no cerrado brasileiro.

Apesar de não ter evidenciado um padrão espacial tão forte quanto aquele observado para a área, o correlograma para o peso do corpo demonstrou que as três primeiras classes apresentaram índices I de Moran significativos positivos, seguidas da sétima e décima classes para as quais os índices obtidos foram significativos e negativos (as outras classes não apresentaram índices I de Moran significativos).

O mapeamento do padrão espacial do peso do corpo e da área de distribuição geográfica dos mamíferos do cerrado foi efetuado pela interpolação dos dados através do algorítimo DWLS (distance weighted least-squares) e evidenciou uma tendência longitudinal para as duas variáveis, principalmente no sentido sudestenoroeste (Fig. 6). 


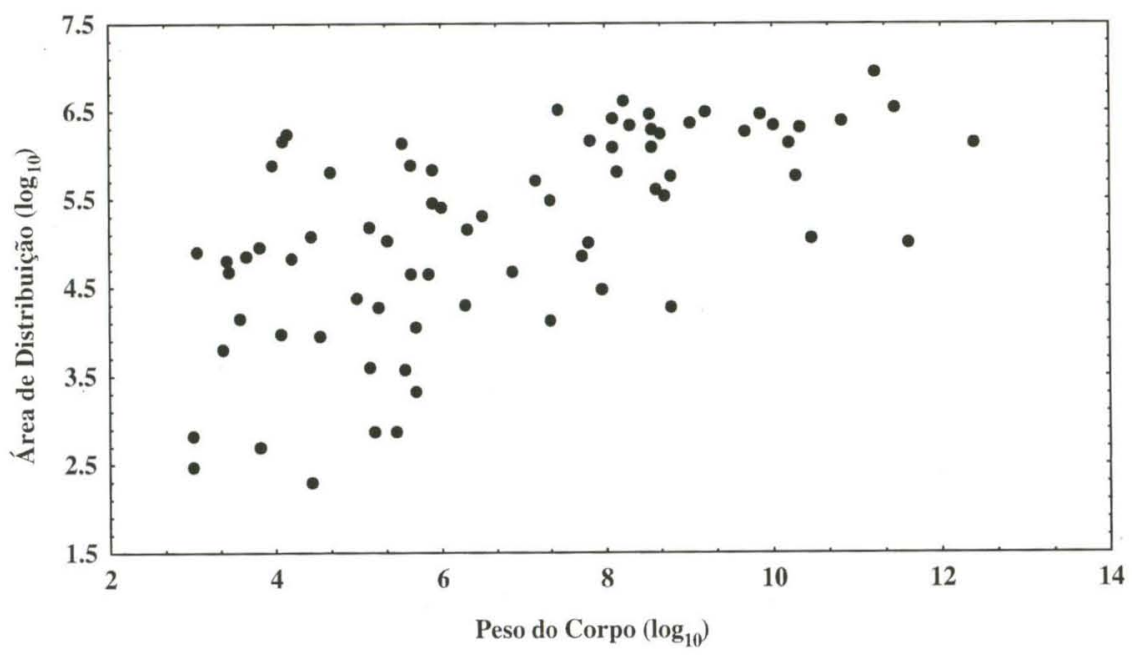

Fig. 4. Relação entre o peso do corpo e a área de distribuição geográfica, para os dados logaritmizados, de 80 espécies de mamíferos com ocorrência na região do cerrado brasileiro.

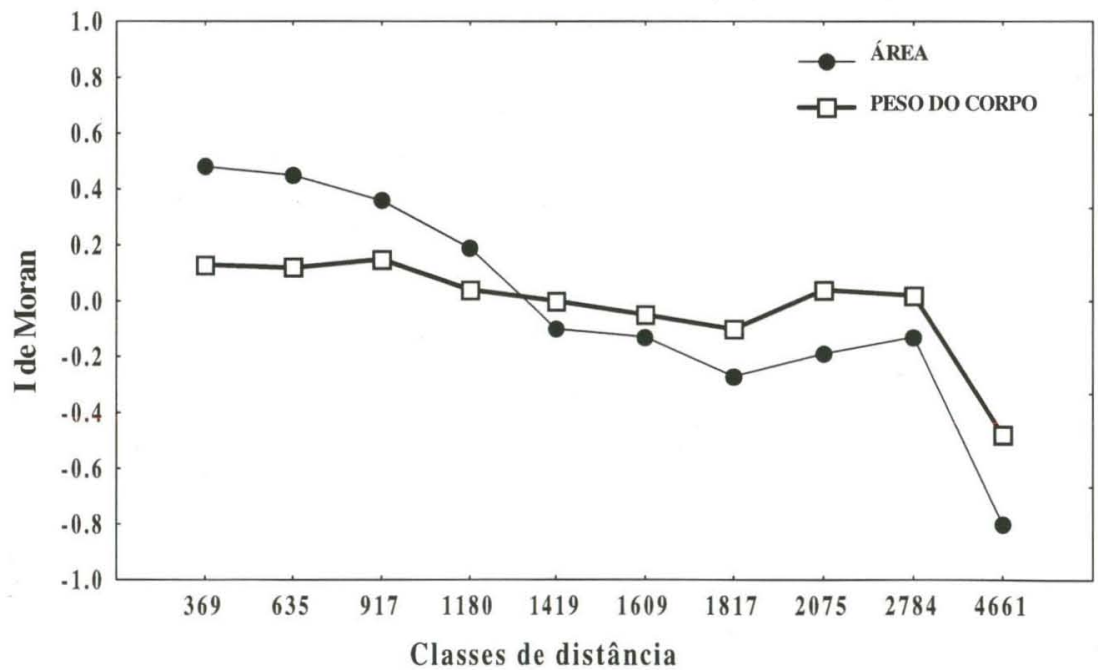

Fig. 5. Correlogramas espacias do peso do corpo (linha espessa) e da a área de distribuição geográfica (linha fina), para 80 espécies de mamíferos com ocorrência no cerrado brasileiro.

O padrão da área de distribuição foi semelhante àquela obtida para o peso. As espécies com áreas de distribuição variando entre pequena e média apresentaram os seus pontos médios concentrados na região mais central do cerrado. Contudo, aquelas com as áreas de distribuição maior tenderam a se distribuir ao longo do eixo longitudinal, em sentido noroeste (Fig. 7). 


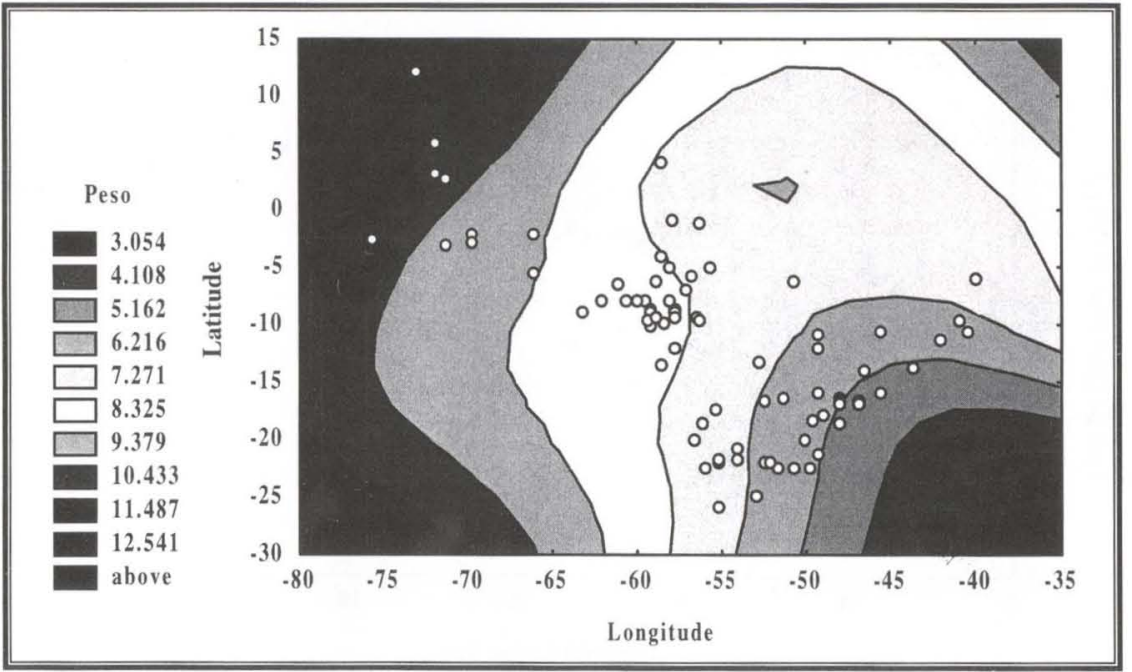

Fig. 6. Padrão espacial do peso do corpo de 80 espécies de mamíferos com ocorrência na região do cerrado brasileiro, interpolado pelo algorítimo DWLS ("distance weighted least-squares").

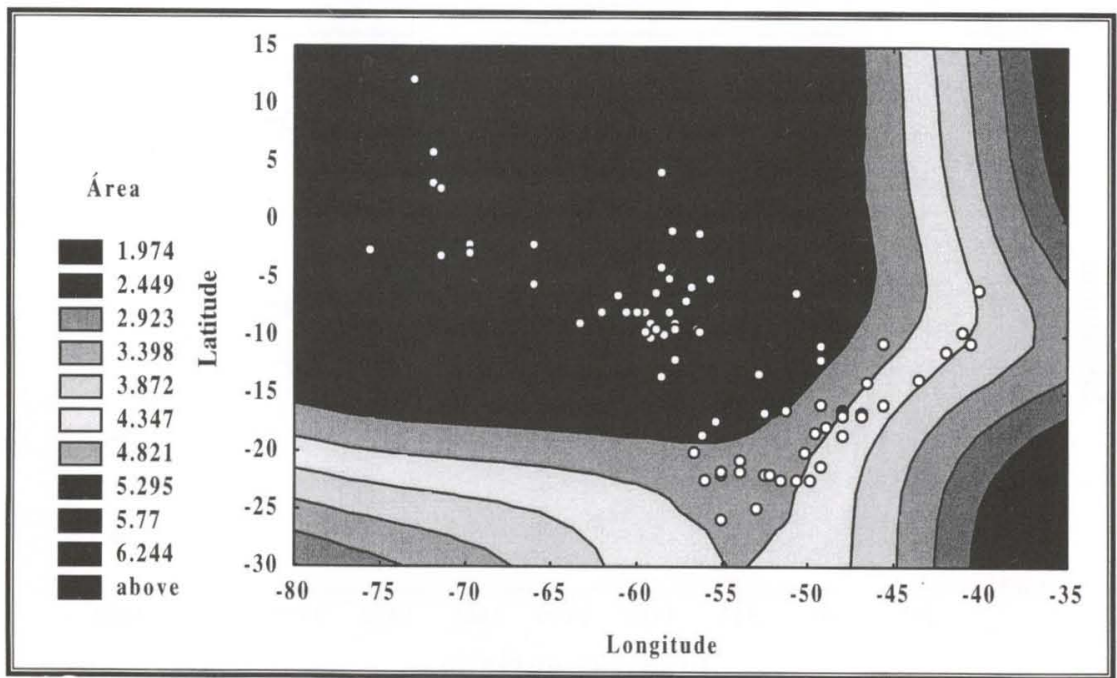

Fig. 7. Padrão espacial da área de distribuição geográfica de 80 espécies de mamíferos com ocorrência na região do cerrado brasileiro, interpolado pelo algorítimo DWLS ("distance weighted least-squares").

Os efeitos espaciais detectados pela análise de autocorrelação e pelo DWLS foram removidos através de uma análise de superfície de tendência (AST) de primeira ordem (linear), em conjunto com uma análise de variância (ANOVA) para as ordens de mamíferos, combinadas em um único modelo generalizado. 
Combinando a AST-Linear e a ANOVA, foram analisados simultaneamente os efeitos espaciais e taxonômicos para as variáveis. Observou-se que $61 \%\left(\mathrm{R}^{2}\right)$ da soma dos quadrados totais da área de distribuição geográfica foi explicada pela posição espacial e por um efeito taxonômico (Fig. 8). A hipótese conjunta de que todos os coeficientes de regressão são iguais a zero foi rejeitada para a análise da área $(\mathrm{F}=14,387 ; \mathrm{P}<0,01)$. Em relação ao peso do corpo, observou-se que $69 \%$ $\left(\mathrm{R}^{2}\right)$ da sua soma de quadrados totais está sendo explicada por efeitos espaciais e taxonômicos e a hipótese conjunta de que todos os coeficientes de regressão são iguais a zero também foi rejeitada $(F=19,034 ; \mathrm{P}<0,01)$. Entretanto, como indicado pela partição da soma de quadrados do modelo generalizado (Tab. II) e pelo correlograma (Fig. 5), o tamanho do corpo está menos estruturado espacialmente do que a área, de modo que o $\mathrm{R}^{2}$ do peso pode ser atribuído aos efeitos taxonômicos, enquanto que para a área pode ser observado o efeito inverso.

A relação entre os resíduos do modelo para as variáveis se manteve positiva e significativa $(\mathrm{r}=0,32 ; \mathrm{P}<0,05)$. Assim, a relação entre o peso do corpo e área de distribuição dos mamíferos do cerrado pode ser explicada por processos ecológicos e evolutivos, que estão se expressando mesmo na ausência das perturbações causadas pelos efeitos de autocorrelação espacial e filogenética (Fig. 9).

Tabela II. Partição da soma de quadrados do modelo generalizado para as variáveis área de distribuição e peso do corpo (em escala logaritmica) de 80 espécies de mamíferos com ocorrência no cerrado, em relação aos efeitos espaciais e taxonômicos ( $R^{2}$ indica o ajuste do modelo para cada variável).

\begin{tabular}{lcc}
\hline \multicolumn{1}{c}{ Efeito } & Área $(\%)$ & Peso $(\%)$ \\
\hline Padrão espacial & 60,110 & 30,590 \\
Grupo taxonômico & 39,880 & 69,410 \\
\hline $\mathrm{R}^{2}$ & 0,608 & 0,691 \\
\hline
\end{tabular}

\section{DISCUSSÃO}

O padrão de distribuição assimétrica à direita observado para a variável peso do corpo não seguiu uma distribuição normal, mesmo após a logaritmização, como observado para mamíferos e aves da América do Norte (BROWN \& MAURER 1987, 1989; BROWN 1995). Essa variável apresentou uma forma especial de distribuição (desviada da distribuição normal), onde se observa um deslocamento do peso modal (curtose significativa) à esquerda (BRowN 1995). A área também apresentou uma distribuição assimétrica, o que deve garantir a forma poligonal do espaço em envelope (BROWN \& MAURER 1987, 1989; TAYLOR \& GOTELli 1994; GASTON \& BLACKBURN 1996a; BONFIM et al. 1998).

A análise macroecológica das variáveis peso do corpo e área de distribuição geográfica para as espécies de mamíferos do cerrado revelou um padrão que corrobora o modelo sugerido por Brown (BROWN \& MAURER 1987, 1989; BROWN 1995), onde se observa uma relação positiva entre as duas variáveis (BROWN 1995). Essa relação pode ser definida por um espaço poligonal, limitado por linhas de 


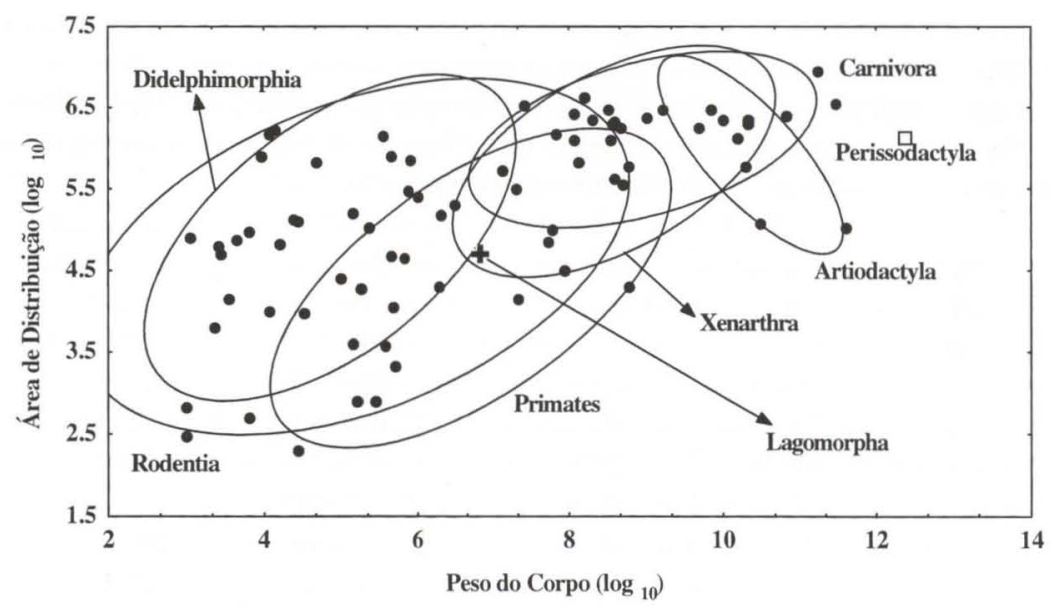

Fig. 8. Estruturação ao nivel taxonômico da ordem na relação área de distribuição geográfica/peso do corpo, para os oito grupos analisados de mamíferos com ocorrência no cerrado brasileiro.

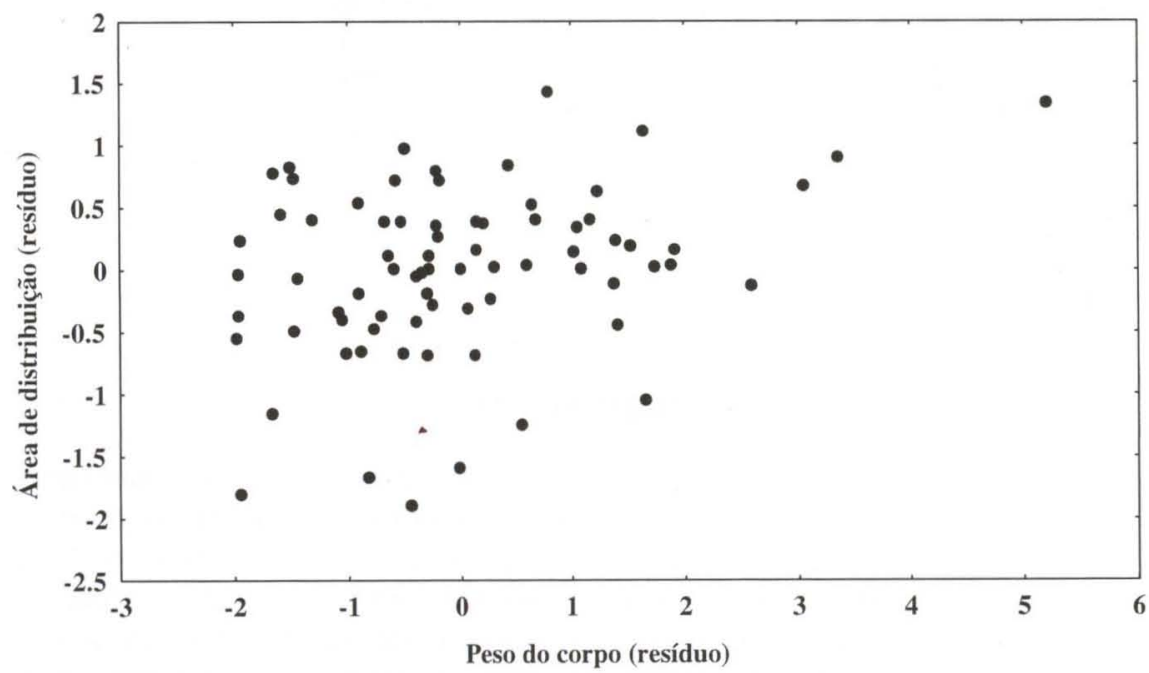

Fig. 9. Relação entre os residuos do modelo generalizado para efeitos espaciais e taxonômicos para as variáveis macroecológicas peso do corpo e área de distribuição geográfica, de 80 espécies de mamíferos com ocorrência no cerrado brasileiro.

restrição ("constraints") físicas, ecológicas e evolutivas. Assim, espécies maiores tendem a apresentar áreas extensas de distribuição, como discutido previamente na introdução. Os resultados observados nesse trabalho reforçam a idéia de que essa correlação pode ser um padrão biogeográfico geral. Esse padrão de correlação positiva foi observado para outros grupos taxonômicos analisados em grandes 
escalas, tais como os peixes do gênero Cyprinella (TAYLOR \& GoTELLI 1994), mamíferos e aves da América do Norte (BROWN \& MAURER 1987, 1989), Anseriformes analisados em escala global (GASTON \& BLACKBURN 1996a), além de serpentes viperídeas e corujas da América do Sul (BONFIM et al. 1998; SANT'ANA \& DiNIZ-FILHO 1999).

Padrões espaciais ao longo de gradientes latitudinais têm sido usualmente considerados para explicar a distribuição dos organismos, em termos da variação no tamanho do corpo (regra de Bergmann), na área de distribuição (regra de Rapoport) e na própria riqueza de espécies. Muitos autores demonstraram uma correlação positiva entre a área de distribuição geográfica e a latitude (regra de Rapoport) em grupos taxonômicos do Velho e do Novo Mundo (STEVENS 1989; RUGGIERO 1994; LYONS \& WILLIG 1997; GASTON et al. 1998). RUGGIERO (1994), analisando separadamente táxons de mamíferos da América do Sul encontrou uma correlação positiva entre a área de distribuição e a latitude, em concordância com a regra de Rapoport. Entretanto, o padrão espacial observado para os mamíferos do cerrado não mostrou nenhuma tendência latitudinal evidente; ao contrário, foi observada uma tendência longitudinal que pode estar refletindo um efeito de continentalidade na distribuição desse grupo (LYONS \& WILLIG 1997). Assim, a distribuição dos mamíferos do cerrado pode estar respondendo, de maneira significativa, à forma do continente Sul Americano. Esse aparente efeito de continentalidade parece ocorrer em outros grupos taxonômicos, como para as serpentes da família Viperidae analisadas na região neotropical (BONFIM et al. 1998). Foi observado, para os mamíferos do cerrado, que espécies com peso maior, e consequentemente com grandes áreas de distribuição (modelo de Brown), se apresentam distribuídas nas porções mais extensas, em longitude, da região neotropical, seguindo uma tendência longitudinal que coincide com a possibilidade física de distensão das áreas de distribuição das espécies. (RUGGIERO 1994; GASTON et al. 1998; BONFIM et al. 1998; MARQUET \& TAPER 1998).

LYONS \& WILLIG (1997) sugerem que a não apresentação de um padrão latitudinal claro para a área de distribuição geográfica pode não ser uma discordância total à regra de Rapoport. Esses autores, demonstraram através de simulações sob um modelo nulo, que apesar da diferença na forma do continente Sul Americano em relação à América do Norte (o que explicaria uma correlação negativa entre a área e a latitude, pois na região dos trópicos a América do Sul se alarga, enquanto que a América do Norte se estreita), o "espírito da regra de Rapoport" ainda poderia estar sendo mantido. Aparentemente, o tamanho das áreas de distribuição das espécies de mamiferos analisados por eles (marsupiais e quirópteros) aumentou em direção aos trópicos menos do que o esperado para um modelo nulo associado à forma do continente sul-americano. Dessa forma, mecanismos determinísticos, tais como a relação da área de distribuição com a tolerância da espécie à variação climática latitudinal (regra de Rapoport), ainda poderiam estar explicando esse padrão de distribuição dos mamíferos da América do Sul.

Os resultados do modelo generalizado demonstraram a influência diferenciada dos efeitos taxonômicos e espaciais sobre o peso do corpo e sobre a área de distribuição geográfica. O peso do corpo dos mamíferos do cerrado está significa- 
tivamente mais estruturado ao longo da filogenia (HARVEY \& PAGEL 1992) do que ao longo do espaço, como foi sugerido pelos resultados da ANOVA a nível de ordem. É provável que uma análise mais refinada, utilizando métodos comparativos baseados em uma filogenia mais completa e consensual dos mamíferos possa demonstrar de maneira mais precisa esses efeitos de enércia filogenética (CHEVERUD et al. 1985; HARVEY \& PAGEL 1992; Diniz-FilHo et al. 1998). A área de distribuição, entretanto, não está sofrendo um efeito significativo de ancestralidade, estando porém respondendo de maneira significativa a um efeito de posição espacial.

$\mathrm{O}$ aspecto mais importante desse trabalho foi demonstrar que os padrões macroecológicos observados para mamíferos com ocorrência na região do cerrado, quando mensurados a partir de variáveis tais como peso do corpo e área de distribuição, estão em concordância com aqueles observados para outros grupos, em diversas regiões do mundo (BROWN \& MAURER 1987, 1989; TAYLOR \& Gotelli 1994; GASTON \& BLACKBURN 1996a; BonfiM et al. 1998). Há uma relação positiva definida como um envelope, mesmo depois de extraídos os efeitos de autocorrelação espacial e filogenética, o que descarta as explicações (2), (3) e (4) propostas por GASTON \& BLACKBURN (1996b) para essa relação. Assim, para os mamíferos neotropicais com ocorrência na região do cerrado, uma análise macroecológica concorda com o modelo sugerido por BROWN \& MAURER $(1987,1989)$, onde a correlação entre o peso do corpo e área de distribuição geográfica se apresenta positiva independentemente da localização espacial das espécies e do grupo taxonômico ao nível da ordem

AGRADECIMENTOS. À Fernanda Silva Bonfim, Leandro Silveira, Anah Tereza de Almeida Jácomo e Carlos Eduardo Ramos de Sant'Ana, pelas valiosas discussões sobre métodos quantitativos em Biologia omparada, Ecologia e Evolução dos mamíferos da américa do Sul. Somos especialmente gratos a Luis Maurício Bini por suas avaliações e sugestões estatísticas e, também aos Professores Dr. Sérgio Furtado dos Reis e Dr. Fabrizio D’Ayala Valva. Nosso programa de pesquisa em Ecologia e Evolução é apoiado pelo CNPq, CAPES, FUNAPE/PRPPG-UFG e UEG.

\section{REFERÊNCIAS BIBLIOGRÁFICAS}

ALHo, C.J.R., 1993. Distribuição da fauna num gradiente de recursos em mosaico, p. 213-262. In: M.N. PINTO (Ed.). Cerrado: caracterização, ocupação e perspectivas. Brasília, Editora Univ. Brasília, $2^{\mathrm{a}}$ Edição, 681p.

ARITA, H.T.; J.G. RoBINSON \& K.H. REDFORD. 1990. Rarity in neotropical mammals and its ecological correlates. Conserv. Biol. 4: 181-192.

Blackburn, T.M. \& K.J. Gaston. 1998. Some methodological issues in macroecology. Amer. Nat. 151: 61-83.

BonfIM, F.S.; J.A. DinIZ-Filho \& R.P. BASTOS. 1998. Spatial patterns and the macroecology of South American viperid snakes. Rev. Brasil. Biol. 58: 97-103.

Brown, J.H. 1995. Macroecology. Chicago, Univ. Chicago Press, XII+269p.

BROWN, J.H. \& B.A. MAURER. 1987. Evolution of species assemblages: effects of energectic constraints and species dynamics on the diversification of North America Avifauna. Amer. Nat. 130: 1-17.

. 1989. Macroecology: the division of food and space among species on continents. Science 243: $1145-1150$. 
Campbell, J.A. \& W.W. Lamar. 1989. The venemous reptiles of Latin America. Chicago, Cornell Univ. Press, 414p.

Cheverud, J.M.; M.M. Dow \& W. Leutenegger. 1985. The quantitative assessement of phylogenetic constraints in comparative analyses: sexual dimorphism in body weight among primates. Evolution 39: 1335-1351.

Diniz-Filho, J.A. \& R. Balestra. 1998. Hierarchical effects on body size evolution and the macroecology of South American rainforest mammals. Ecol. Austral 8: 23-30.

Diniz-FiLHo, J.A. \& H. FowLER. 1998. Honey ants (Genus Myrmecocystus) macroecology: effect of spatial patterns on the relationship between worker body size and geographic range size, Envir. Ent. 27 (5): 1094-1101.

Diniz-FiLho, J.A. \& C.M. VieirA. 1998. Padrões e processos na evolução do tamanho do corpo em carnivoros (Mammalia) da América do Sul. Rev. Brasil. Biol. 58 (4): 649-657.

EISENBERG, J.F. 1989. Mammals of the neotropies: the northen neotropics. Chicago, Univ. Chicago Press, $\mathrm{X}+449 \mathrm{p}$.

Emmons, L.H. 1997. Neotropical rainforest mammals: a field guide. Chicago, Univ. Chicago Press, $2^{\text {nd }}$ ed., XVI $+307 \mathrm{p}$.

Fonseca, G.A.B.; G. Herrmann; Y.L.R. Leite; R.A. Mittermeier; A.B. Rylands \& J.L. Patton. 1996. Lista anotada dos mamiferos do Brasil. Occas. Pap. Conserv. Biol.: 1-38.

GASTON, K.J. 1991. How large is a species geographic range. Oikos 61: 434-438.

. 1996. Species-range-size distribuitions: patterns, mechanisms and implications. Trends Ecol. Evol. 11: 197-201.

Gaston, K.J. \& J.H. LAWTON. 1988a. Patterns in the distributions and abundance of insect populations. Nature 331: 709-712.

-1988b. Insect herbivores on bracken do not support the core-satellite hypothesis. Amer. Nat. 134: 761-777.

GASTON, K.J. \& T.M. BLACKBURN. 1996a. Global scale macroecology: interations between population size, geographic range size and body size in the Anseriformes. Jour. An. Ecol. 65: 701-714.

. 1996b. Range size-body relationships: evidence of scale dependence. Oikos 75: 479-485.

GASTON, K.J.; T.M. BLACKBURN \& J.I. SPICER. 1998. Rapoport's rule: time for an epitaph?. Tree 13: 70-74.

Harvey, P.H. \& M.D. Pagle. 1992. The comparative method in evolutionary biology. Oxford, Oxford Univ. Press, VII +239p.

LaBarbera, M. 1989. Analyzing body size as a factor in ecology and evolution. Ann. Rev. Ecol. Syst. 20: $97-117$

LEGENDRE, P. 1993. Spatial autocorrelation: trouble or new paradigma? Ecology 74: 1659-1763.

LEGENDRE, P. \& M.J. ForTIN. 1989. Spatial pattern and ecological analysis. Vegetatio 80: 107-138.

LEvin, S. 1992. The problem of pattern and scale in ecology. The Robert H. MacArthur Award Lecture. Ecology 73: 1943-1967.

LYONS, S.K. \& M.R. WILLIG. 1997. Latitudinal patterns of range size: methodological concerns and empirical evaluations for New World bats and marsupials. Oikos 79: 568-580.

Mares, M.A. \& H.H. Genoways. 1982. Mammalian biology in South America. Special Publicantion Series. Pymaturing Laboratory of Ecology. University of Pittsburgh, XII+551p.

MARQUET, P.A. \& M.L. TAPER. 1998. On size and area: patterns of mammalian body size extremes across landmasses. Evol. Ecol. 12: 127-139.

Moojen, J. 1952. Os roedores do Brasil. Rio de Janeiro, Biblioteca Científica Brasileira, Série A-II, Inst. Nacional do Livro, 214p.

ODEN, N.L. 1984. Assenssing the significance of a spatial correlogram. Geogr. Anal. 16: 1-16.

Peters, R.H. 1993. The ecological implications of body size. Cambridge, Cambridge Univ. Press, $\mathrm{XII}+329 \mathrm{p}$.

REDFORD, K.H. \& J.F. EISENBERG. 1992. Mammals of the Neotropics: the southern cone. Chicago, Univ. Chicago Press, IX+430p. 
RICKLEFS, R.E. \& D. SCHLUter. 1993. Species diversity in ecologyeal comunities: historical and geographical perspectives. Chicago, Univ. Chicago Press, 415p.

RUgGiero, A. 1994. Latitudinal correlates of the sizes of mammalian geographical ranges in South America. Jour. Biogeogr. 21: 545-559.

SANT'ANA, C.E.R. \& J.A. Diniz-FilHo. 1999. Macroecologia de corujas (Aves, Strigiformes) da América do Sul. Ararajuba 7 (1): 3-11.

Stevens, G.C. 1989. The latitudinal gradient in geographical range: how so many species coexist in the tropics. Amer. Nat. 133: 240-256.

SOKAL, R.R. \& N.L. OdEN. 1978a. Spatial autocorrelation in biology. I. methodology. Biol. Jour. Lin. Soc. 10: 199-228.

. 1978b. Spatial autocorrelation in biology. II. Some biological implications and four aplications of ecological and evolutionary interest. Biol. Jour. Lin. Soc. 10: 229-249.

SOKAL, R.R. \& F.J. ROHLF. 1995. Biometry. New York, W.H. Freeman and Company, $3^{\text {rd }}$ ed., $\mathrm{XIX}+887 \mathrm{p}$.

TAYLOR, C. M. \& N.J. GotelLI. 1994. The macroecology of Cyprinella: correlates of phylogeny, body size and geographic range. Amer. Nat. 144: 549-569.

TOKESHI, M. 1993. Species abundance patterns and community structure. Adv. ecol. Res. 24: 111-186.

WARTENBERG, D. 1989. SAAP: Spatial autocorrelation analysis program. New York, Exeter Publication Company, 29p.

WILKINSON, L. 1989. Systat/Sygraph: the system for statistics. Systat. Inc., Evanston, IL.

WILSON, D.E. \& D.M. REEDER. 1993. Mammal species of the world: a taxonomic and geographic reference. Washington, Smithsonian Inst. Press, $2^{\text {nd }}$ ed., $1207 \mathrm{p}$.

Recebido em 11.III.1999; aceito em 07.XII.2000. 